\title{
How the excitation system parameters and the generator protection settings affect the reliability of electricity delivery from distributed generation facilities
}

\author{
Pavel Ilyushin ${ }^{1 *}$, and Aleksandr Kulikov² \\ ${ }^{1}$ Energy Research Institute of Russian Academy of Sciences, Nagornaya St., 31, bld. 2, Moscow, Russia \\ ${ }^{2}$ Nizhny Novgorod state technical university n.a. R.E. Alekseev, Minin St., 24, Nizhny Novgorod, Russia
}

\begin{abstract}
The paper analyzes the causes of disruptions in electricity delivery to critical users in power areas that operate distributed generation facilities (DG), hereinafter referred to as DG areas. Statistics is shown to be affected by the types of the excitation systems (ES) chosen for the DG generators, as well as by the relay protection (RP) settings as configured when designing the facilities. The paper dwells upon the specifications of the ES's used in low-power generators, their effective applications, as well as the consequences of disturbances in the power area or in an external grid. It is proven herein that RP settings as configured by generator manufacturers are often suboptimal, which jeopardizes their further operation, prevents the operator from aligning these settings to those of the grid RPs, and results in unnecessary disconnections. The paper also details upon calculating the parameters of DG-enabled grids in the common software suits, as well as on how to make a list of effective contingencies. It further gives recommendations on grouping the calculation problems by initial disturbance to optimize the number of projected scenarios. The authors prove that ES selection and generator RP configurations must be appropriate if DG facilities are to deliver electricity reliably.
\end{abstract}

\section{Introduction}

Statistics of enterprise-level disruptions in electricity delivery in DG areas is analyzed herein, and the finding is that appropriate design for ES parameters and RP settings is important for reliable electricity delivery.

Cases of industrial DG areas being disconnected from the grid due to a short SC-induced voltage sag are reported on a regular basis. The gravest situations occur when a power area is only powered by a single power transmission line, and the external grid is switched to maintenance mode. In that case, DG facilities either end up being unable to reliably cover the load in the area, which results in disconnecting it; or are disconnected by the RP devices triggered by undervoltage [1-3].

Reason \#1 is that ES's were selected inappropriately when designing the area's electricity system. Reason \#2 is that a voltage sag might be longer or deeper than what the generator RPs are designed for. The consequences can be grave if that results in disconnecting life support systems or continuous production processes $[4,5]$.

There are several ES types that have some fundamental differences that must be borne in mind when integrating DG facilities in local grids, as well as some small differences that have little to no effect on generator operations. Depending on the operating situation and which electric devices are connected to the areal grid, some ES properties will be decisive while others will be insignificant.
Generators by foreign manufacturers use ES's designed in compliance with their respective national or common European standards. At the same time, it is the ES algorithms and settings that determine voltage quality in the area, the stability of generators exposed to external disturbances, the parameters of transients, abnormal, and emergency operations.

The paper describes generator ES's widely used at DG facilities and details their operating principles as well as how to approach RP configuration from the standpoint of consumers' quality and reliability requirements.

\section{Specifics of generator excitation systems}

Let us discuss the commonly used DG generator ES types listed here by reliability for consumers in ascending order, and analyze their features:

- SHUNT is used at generators up to $150 \mathrm{kVA}$ or even up to $500 \mathrm{kVA}$ in certain cases; it is designed to control generators only in steady state, and it does not support continuous voltage control.

Since SHUNT is powered by the generator busbars, it can maintain present excitation current or generator voltage (reactive power; power factor) in steady states when the voltage, the power, and the current are within the acceptable limits. This is effective when one needs to

* Corresponding author: ilyushin.pv@mail.ru 
optimize the local parameters, e.g. to minimize the losses, and the area has other parameter control tools.

SHUNT shows its vulnerability when the generator busbars have a voltage sag, i.e. when the external or internal grid short-circuits, the automated reclosing is unsynchronized, etc. In case of lack of voltage control, the generator excitation current decreases proportionally to the busbar undervoltage, which prevents the operating parameters from stabilizing. Whether SHUNT-equipped generators are suitable must be checked by calculating the parameters for specific operating situations.

- PMG, or permanent magnet generator, differs from SHUNT in the sense that the ES has a power source of its own; it is a rotary exciter that is independently subexcited by permanent magnets, so the excitation current does not depend on the generator busbar voltage; this reduces the risks of busbar voltage sags having negative impact.

Figure 1 shows the calculated transients for a 3-phase $\mathrm{SC}\left(t_{\mathrm{sc}}=0.18 \mathrm{~s}\right)$ in an area that has a DG facility weakly connected to the external grid; the calculations are done for SHUNT- and PMG-equipped generators. The SC reduces the voltage below the generator RP setting $(U \leq$ $\left.0.8 U_{\text {nom }}\right)$ for $>1.2 \mathrm{~s}$ in case of SHUNT and $>0.8 \mathrm{~s}$ in case of PMG, see Figures $1 \mathrm{a}$ and $1 \mathrm{~b}$, respectively; the reason is the motors self-starting.

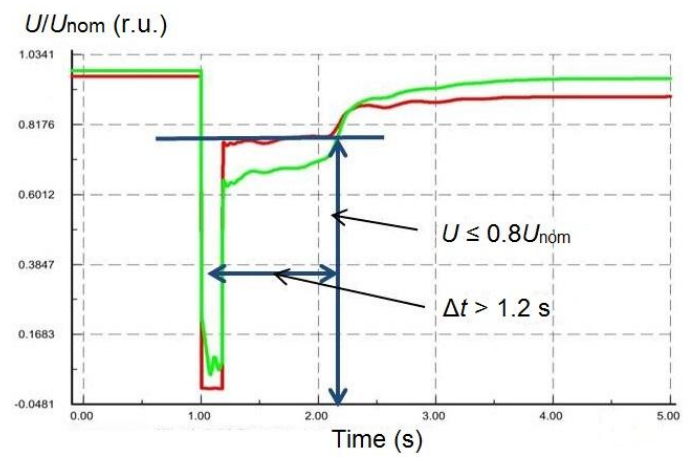

a)

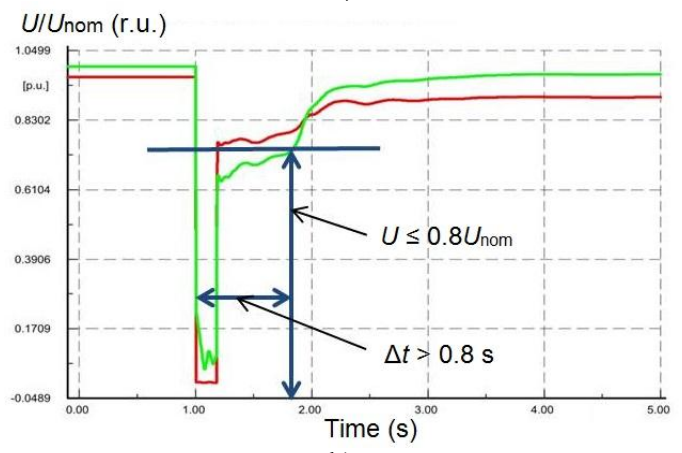

b)

Fig. 1. 3-phase SC transient: (a) SHUNT-equipped generators; (b) PMG-equipped generators.

If the generator RPs are configured to be voltagetriggered after $\sim 1 \mathrm{~s}$, SHUNT will disconnect them while PMG will not.

- AREP (patented by Leroy-Somer) performs similarly to PMG as shown by transient calculations; it somewhat stabilizes the load by increasing the excitation current as the stator current rises (it implements compounding by stator current),

- Analog or digital automatic voltage regulator provided upon the Customer's request.

These regulators are used with low-power generators and differ in terms of response latency as well as in what parameters can be used in the control law. Important factors include the maximum excitation current and the rate at which it builds up, which determines how fast the operating parameters are stabilized after the disturbance has been addressed.

- A specialized automatic voltage regulator with additional functionality to improve the parameters of transients in load surging and shedding.

Possible regulation options include:

- U/f ratio to enable nearly instant generator voltage rise after a busbar voltage sag;

- Load Agreement Module to provide a more complex control law that could be adaptively configured for load surges exceeding $60 \%$ of $P_{\text {nom. }}$.

These types of regulation are most important for generators operating in insular energy systems (herein referred to as 'power areas', 'areas', and such) or when a power area is islanded by the grid. Analysis of such controls shows that:

- Reducing the voltage of a generator driven by an internal combustion engine (ICE) by means of a Load Agreement Module is an effective method for reducing dynamic frequency drops when the generator is facing a load surge provided that the active load magnitude is essentially dependent on the voltage,

- Voltage reduction might not have a positive effect if the load does not (significantly) depend on the voltage, which is typical of load sites where most active power is used by motors,

- If the load power is switching without a dead time as is the case for triggering automatic load transfers (ALT), this jeopardizes further self-starting and starting of asynchronous motors as the voltage sags,

- When the Load Agreement Module undervolts the generator, this undervoltage can be unacceptable if it disconnects the electricity users and triggers a voltage collapse in the area $[6,7]$.

Thus, before commissioning generators that have a Load Agreement Module in their ES's, one must analyze the transient parameters to confirm that the function is suitable for their specific operating conditions.

Leroy-Somer lists the following key applications for ES types: SHUNT is for basic power backup and telecommunications; PMG and AREP is for maritime industries, construction, hospitals, banks, and electricity generation. Note that delivery electricity to industrial facilities that carry multiple motors is not listed as a suitable application for these ES's.

Besides, PMG and AREP also allow the stator current to reach the triple value of $I_{\text {nom }}$ for 10 seconds, which is due to free currents in the SC current rather than due to forcing an excitation.

Thus, SHUNT, PMG, and AREP all have the same effective application: steady state, no overload, stable busbar voltage. Short-term undervoltage due to external disturbances will have the highest chance of disabling a 
SHUNT generator and the lowest chance to disable an AREP generator.

Transient calculations show that reliable electricity delivery to industrial users from SHUNT, PMG, or AREP-equipped generators is impossible in most operating situations where a disturbance occurs, whether it is within a standard tolerance range or (and especially) exceeds it.

Regulation algorithms for steady states and transients alike can be implemented in excitation systems equipped with an analog or digital automatic voltage regulator or a specialized automatic voltage regulator with additional functionality.

\section{Configuring the generator RPs}

To protect generators from drastic fluctuations in operating parameters, manufacturers tend to configure RPs in such ways as to significantly narrow the acceptable operating range, which prevents these protections from running normally.

Generator disconnection reasons are associated with their tendency to be ever more efficient and costeffective given that emergencies are being handled ever quicker, and the post-emergency parameters stabilize at ever greater rates. However, operators fail to bring the generator RP settings in line with those of the grid elements, resulting in unnecessary disconnections $[8,9]$.

Non-selective generator disconnections at DG facilities disrupt electricity delivery to consumers and cause load surges in the adjacent grid, overloading it. One important consideration here is that generator RPs cannot be reconfigured without the manufacturer's permit until the warranty expires, and unauthorized reconfiguration renders the warranty null and void.

Consider a gas-piston power plant (GPPP) that comprises $4 * 2.4$ MW generators configured to disconnect if in all the three phases, the voltage stays above $110 \%$ or below $90 \% U_{\text {nom }}$ for 0.2 seconds. How specifically a transient goes depends to a great extent on what comprises the load and on the resulting stability of AC motors. As a GPPP that carries an industrial-grade load becomes islanded with a $15 \%$ active power deficit, it causes all the generators to disconnect, and the electricity delivery is disrupted, see Figure 2.

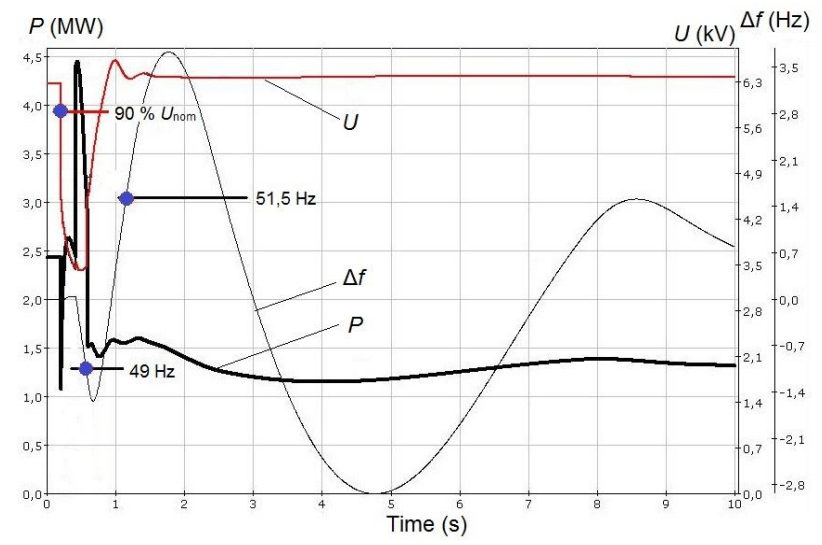

Fig. 2. Transient: GPPP loaded, islanded.
Figure 2 shows RP settings, namely $U$ sag and $f$ deviation thresholds; the RPs are off. As can be seen there, the transient would be satisfactory if the RPs did not disconnect the generators.

Configuring the generator RPs to be triggered by undervoltage without monitoring the currents is not advisable, as GS windings can only be damaged by overheating if the stator/rotor current overloads are longer or stronger than permissible $[10,11]$.

The permissible magnitudes of currents are tailored by the manufacturers specifically to the design of the generators, primarily to the thermal endurance class (temperature index) of its windings in correlation with the temperatures, cooling system type, and coolant used with the stator and rotor windings.

In case the stator current exceeds the permissible duration or magnitude, RP disconnects the generator from the external grid; if the rotor current does the same, RP lowers the excitation current (de-excites) to a threshold, below which the rotor windings cannot dangerously overheat.

Besides, when configuring generator RPs, it should be borne in mind that the actual sag duration $U \sim$ is twice as long, especially in case of a three-phase SC, as SCshut motors demand greater current to return back to their normal rotation speeds. Figure 3 shows a transient where the post-SC voltage sag is longer due to the motors self-starting at the load nodes.

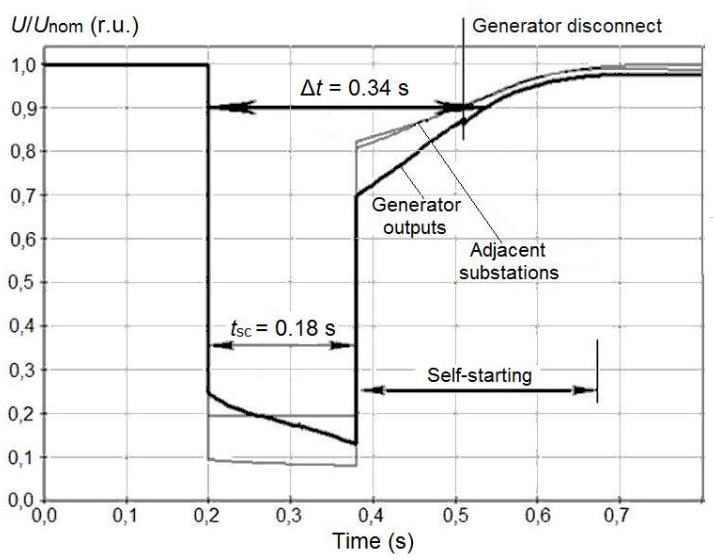

Fig. 3. Three-phase SC transient with motor self-starts.

Importantly, generators can be disconnected by RPs triggered by frequency deviations $\left(f<f_{\min }\right.$ and $\left.f>f_{\max }\right)$, which are designed with the following in mind:

- Mechanical speed constraints (mechanical strength),

- Avoiding getting too close to resonant frequencies, as those greatly amplify the vibrations,

- Avoiding rotation speed drops to prevent failures or inappropriate functioning of the auxiliary equipment designed to enable the generator drive to function properly, e.g. when the air-and-fuel mixture fails to ignite properly in the ICS cylinders.

Significant load surge or shedding not associated with an SC may also result in disconnecting the generators in islanded operation, which can happen when connecting or disconnecting high-power users or their groups. Generator manufacturers specify maximum 
acceptable voltage surges that depend on the initial generator load and will not cause the RPs to initiate disconnection routines.

In this case, it is advisable to use process automations that will sequentially start small motor groups while controlling their voltage; the whole procedure must be adjusted to the processes of the facility or to the softstarter (variable frequency drive) of the most powerful motors.

If the generator voltage busbars are connected directly or through the power transformer to an extensive 6-35 kV grid, where time-selective overcurrent protections are used primarily or as backups, the generators have a far higher probability of disconnecting unnecessarily.

Thus, when designing DG areas, it is important to rigorously analyze the transient calculations to find out whether specific generator types (ES parameters and RP settings) are suitable for reliable electricity delivery from DG facilities to the users.

\section{DG-equipped grids: specifics of parameter calculation}

This analysis must be carried out for normal and abnormal operation. If a generator disruption will damage the consumers, the effective contingency list must be made by calculating the parameters of all possible and hazardous operating situations [12-14].

For such calculations, one should bear in mind two specific features of software suites in use:

- Some of the suites have simplified descriptions of the systems that regulate the current power reachable by the steam turbine engines, which is not suitable for simulating gas-turbine engines and ICEs unless adjusted,

- Suites are mainly intended for designing backbone grid infrastructure where transients depend little on whether the load models are correct; however, this is crucial for power areas with DG facilities.

When making the calculation model and running the calculations, focus should be made on:

- Accuracy of the parameters of the grid and power plant (DG facility) equivalent circuits for the power area under consideration as well as for the adjacent sections of the grid,

- RP configurations in the adjacent grid: one needs accurate data on how long an SC the power area and the adjacent grid may have,

- Parameters of the AC motors, the list of which will depend on which software is used, which processes are in place, the motor voltage and installed capacity, as primary motors should be added to the model one by one while others can be generalized by equivalence,

- Statistics on single-phase and multiphase SCs of varying duration, which serves as auxiliary data to help get an idea of disturbance probabilities and their consequences,

- Settings of the process automations that control the core processes. These details are needed to check the settings for consistency with the generator RP settings to prevent unnecessary generator disconnections and minimize the damage associated with disruptions in electricity delivery.

The key factor that makes it difficult to calculate the transients in DG areas consists in the variety of calculation scenarios to be covered, namely:

- The original circuitry of the area and its repair varieties, as the key factor is whether there are any repair varieties and how many of them are there, or the lack of connections to the grid,

- The disposable generation capacity for the current circuitry of the area,

- Features of the DG facility generators: ES parameters, RP settings and time offsets; generator drive power control laws, the regulation principles and the delays of power gain,

- Load magnitudes and variations of what comprises them if such variations are significant (maximum to minimum in case the placement within the area differs),

- Standard and above-standard disturbance application spots, taking into account statistics,

- Peculiarities of RP triggers in the local grid, most importantly the maximum durations of multiphase SCs,

- Other specific features of the power area, which, among other things, may appear in future circuits.

If the calculated transients end in a failure, i.e. some of the electrical equipment is and remains disconnected, some of the motors trip and are disconnected by the RPs, cost-effective contingencies must be in place, such as:

- Automated emergency response systems, the algorithms and configurations of which must be in line with those of the process protections,

- Increasing the installed capacity of the DG facilities,

- Installing an energy storage unit, etc.

When selecting contingencies, it should be borne in mind that normally, the calculation covers not the entire transient (from the initial disturbance to getting back to normal operation) but only its onset that detects the equipment that could be affected by the transient, which is what determines the characteristics of abnormal operation.

In case of a large power area, the circuit used for calculations includes only that equipment, the parameters of which may significantly affect the calculation results. This means that any calculation attempt must strive to:

- Simplify the calculations by excluding the basic cases, handling which only requires understanding the nature of the transients, e.g. when generator RPs are triggered by undervoltage in case of a close SC,

- Reduce the number of logical and calculated scenarios by pregrouping the calculation by class (e.g. types of standard and above-standard disturbances) so as to be able to analyze the results and conclude on the effectiveness of the contingencies.

Consider an example of grouping the calculation problems by initial disturbance for a DG area.

1. Normal start of a major motor (it is most important to analyze direct starts to make a list of contingencies, since such starts have a significant impact 
on the grid parameters).

2. Motor groups switching with a dead time for process or electricity-related reasons other than SCs in the local grid, if such switching may occur.

3. SCs in a grid where ALT, automatic reclosers (ARC), etc. have a deadtime. In that case, the calculation needs to find whether motor self-start or automatic voltage-controlled restart is possible and permissible.

The calculations require adjustments for SCs in the local grid as well as in any connected grids, especially low-voltage ones that use time-selective (for long SCs) overcurrent protections as primary protections or backups.

In most cases, two initial disturbance parameters must be set: the voltage sag depth as measured at the generator busbars at the SC onset $\left(U_{0}\right)$, and the SC duration. $U_{0}$ is an easy-to-calculate parameter convenient for comparing the severity of SCs in different operating situations. However, it alone is not enough for any substantiated conclusion on whether generator RPs will be triggered by voltage or not, as until an SC is over, voltage will continue going down because the braking motors will draw increasing current. It is therefore necessary to monitor the entire transient $U(t)$.

Figure 4 shows the calculated parameters of an islanded DG area. This calculation was made to find the conditions, under which all the motors would be able to self-start while the RPs would not disconnect the generators by frequency drop (the frequency threshold $=$ $47.5 \mathrm{~Hz}$, the time offset $=2$ seconds).

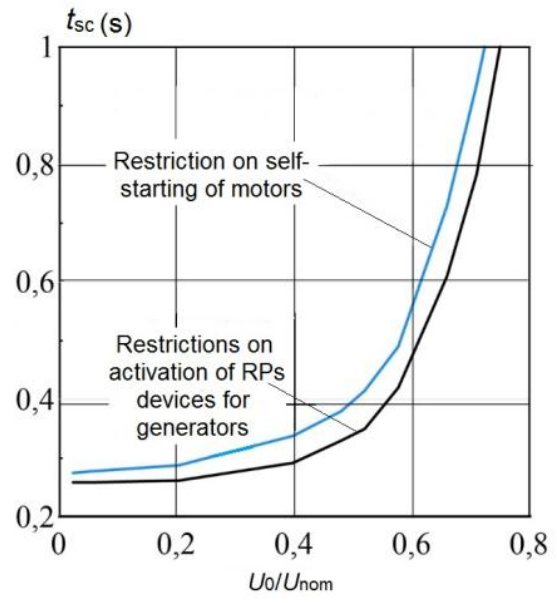

Fig. 4. Permissible SC-induced voltage sag and duration.

Additional calculations should find whether disconnecting non-critical motors that help the more important ones to self-start is permissible, e.g. when reducing the voltage.

\section{Conclusions}

Industrial DG areas regularly report short-term SCinduced voltage sags disrupting electricity delivery to critical users, resulting in an emergency shutdown of the processes, which entails associated damage.

DG facilities commonly use generators that employ excitation systems designed to control only steady states at stable busbar voltage and no overload; they are not designed for continuous voltage control.

It is proven herein that RP settings as configured by generator manufacturers are often suboptimal, which jeopardizes their further operation, prevents the operator from aligning these settings to those of the grid RPs, and results in unnecessary disconnections.

Calculating the transients for DG areas has its own specifics that distinguishes it from similar calculations for backbone grids; this must be borne in mind when designing the system.

If the calculations show that disrupting the generators would jeopardize critical electricity users, then effective contingencies must be placed when designing the area.

For more reliably electricity delivery from DG facilities to users, especially when the area is islanded, the design must provide appropriately selected excitation systems and generator RP settings.

\section{References}

1. B. Buchholz, Z. Styczynski, Smart Grids fundamentals and technologies in electricity networks (Springer Heidelberg, 2014).

2. S. Filippov, M. Dilman, P. Ilyushin, Thermal Engineering, Vol. 66, 12, 869-880 (2019).

3. P. Ilyushin, A. Pazderin, R. Seit, Proceedings 17th International Ural Conference on AC Electric Drives, ACED (2018).

4. M. Schifani, E. Waffenschmidt, R. Iravani, Proceedings International Energy and Sustainability Conference, IESC (2017).

5. K. Kilani, M. Elleuch, A. Hamida, Proceedings 14th International Multi- Conference on Systems, Signals \& Devices, SSD (2017).

6. P. Ilyushin, Proceedings of Methodological Problems in Reliability Study of Large Energy Systems, RSES, Vol. 58 (2018).

7. P. Ilyushin, K. Suslov, Proceedings 2019 IEEE Milan PowerTech (2019).

8. P. Ilyushin, A.Pazderin, Proceedings International Ural Conference on Green Energy, UralCon (2018).

9. S. Budi, A. Nurdiansyah, A. Lomi, Proceedings International Sem. on Intelligent Technology and Its Applications, ISITIA (2017).

10. P. Ilyushin, Power Technology and Engineering, Vol. 51, 6, 713-718 (2018).

11. P. Ilyushin, A. Pazderin, Proceedings 2018 International Conference on Industrial Engineering, Applications and Manufacturing, ICIEAM (2018).

12. Z. Yao, G. Geng, Q. Jiang, IEEE Transactions on Power Systems, Vol. 32, 4, 2704-2713 (2017).

13. P. Ilyushin, O. Sukhanov, Russian Electrical Engineering. Vol. 85. 3. 133-137 (2014).

14. S. Eroshenko, P. Ilyushin, Proceedings 2018 IEEE 59th International Scientific Conference on Power and Electrical Engineering of Riga Technical University, RTUCON (2018). 